\title{
Postoperative bacteriuria, pyuria and urinary tract infection in patients with an orthotopic sigmoid colon neobladder replacement
}

\author{
Katsumi Shigemura, Kazushi Tanaka, Soichi Arakawa, Hideaki Miyake and Masato Fujisawa
}

The purpose of this study is to investigate the prevalence of postoperative bacteriuria, pyuria and urine culture in patients with an orthotopic sigmoid colon neobladder replacement. Urine samples for bacteriuria, pyuria and urine culture, if necessary, were collected at 1, 3, 6, 9 and 12 months after surgery and the presence of blood culture and antibiotic-resistant strains, and their treatments on positive urine culture cases were investigated. Of 209 for bacteriuria and 207 for pyuria urine samples with evaluable data, $95(45.5 \%)$ were positive for bacteriuria and $76(36.7 \%)$ had pyuria (10 or more white blood cells per highpower field). Totally, 30 bacteria were isolated from urine culture of urinary tract infection (UTI) and Klebisiella pneumoniae, Escherichia coli, Staphylococcos aureus and Enterococcus spp. strains were representatively isolated. The incidence of pyuria significantly decreased over time $(P=0.041)$ but that of bacteriuria did not $(P=0.107)$. In them, there were six bacteria $(20.7 \%)$ with antibiotic-resistant strains. The antibiotics used for their treatments representatively were levofloxacin in five cases, tazobactam/piperacillin in three cases and sulfamethoxazole/trimethoprim and cefepime, meropenem in two cases, respectively. In conclusion, these findings suggest that physicians taking care of sigmoid colon neobladder patients need to be aware of these high ratios of bacteriuria, pyuria and UTI, including bacteremia.

The Journal of Antibiotics (2014) 67, 143-145; doi:10.1038/ja.2013.107; published online 30 October 2013

Keywords: bacteriuria; post-surgery follow-up; pyuria; sigmoid colon orthotopic neobladder; urinary tract infection

\section{INTRODUCTION}

Radical cystectomy is a gold standard therapeutic strategy for muscleinvasive bladder cancer without metastasis. ${ }^{1,2}$ Many patients tend to prefer an orthotopic bladder substitution to an ileal conduit or continent urinary reservoir, to achieve normal voiding after cystectomy. ${ }^{3,4}$ Postoperative urinary tract infection (UTI) is a major adverse event that can be caused by bacteiuria or pyuria after diversion. Bacteiuria and pyuria therefore need to be prevented. ${ }^{5}$

There are several methods for neobladder replacement, such as ileal or colon use. The differences in normal flora could be expected to affect postoperative bacteriuria or pyuria. ${ }^{6}$ Suriano et al. ${ }^{5}$ found increased bacteriuria and positive bacterial culture results postoperatively in ileal neobladder and there are numerous reports regarding postoperative bacteriuria after orthotopic neobladder. ${ }^{6}$ Differently from these studies, we focus only on a sigmoid colon neobladder.

In this study, we investigated the prevalence of postoperative bacteriuria, pyuria and UTI, and isolated bacterial cultures for 1 year after sigmoid colon orthotopic neobladder replacement especially focusing on their transitions in a year and the details of UTI cases.

\section{MATERIALS AND METHODS}

\section{Patients}

The study included 56 consecutive outpatients presenting at our university (45 men and 11 women, median age: 67 years and range: $46-79$ years) who previously had a radical cystectomy and sigmoid colon neobladder between 2002 and 2013. All data were collected retrospectively. The patients were evaluated every 3 months including the first month for 12 months.

\section{Evaluation}

Bacteriuria was defined as the presence of at least $10^{5}$ colony-forming units of bacteria per $\mathrm{ml}$ in a urine culture from patients and was shown as $1+\left(10^{5}\right.$ colony-forming units of bacteria per $\mathrm{ml}), 2+\left(10^{6}\right)$ and $3+\left(10^{7}\right.$ or more). Pyuria was defined as the presence of 10 or more white blood cells (WBCs) per high-power field (h.p.f.). ${ }^{7}$ Urinalyses with urinary sediment were performed on every visit to the outpatient department and urine culture tests were performed if the patients have UTI-related symptoms at the time of the visit or on progression of bacteriuria and/or pyuria. Bacterial identification was performed using microscan walk-away system (Siemens Healthcare, Erlangen, Germany) according to the manufacture's protocol.

\section{Susceptibility testing}

Urine was cultured by a semiquantitative method using $1 \mu \mathrm{l}$ of freshly voided midstream urine on $1 \mu \mathrm{l}$ cystine lactose electrolyte-deficient medium agar 
(Nissui Pharmaceutical Co., Ltd., Tokyo, Japan) and $1 \mu \mathrm{l}$ on blood agar (Becton Dickinson, Franklin Lakes, NJ, USA).

\section{Statistical analyses}

Statistical analysis was conducted using linear regression analysis with the PASW Statistics 17.0 software package (for Windows; SPSS Inc., Chicago, IL, USA). The frequency of the occurrences of bacteriuria and pyuria was analyzed with linear regression analysis. Statistical significance was established at the 0.05 level.

\section{RESULTS}

There were 45 male and 11 female patients who underwent radical cystectomy and orthotopic sigmoid colon neobladder reconstruction because of invasive bladder cancer. Median age was 67 (range: 49-79) years. Diabetes mellitus was seen in seven $(12.5 \%)$ patients.

Table 1 Change of bacteriuria after an orthotopic sigmoid colon neobladder replacement

\begin{tabular}{lccccccccc}
\hline & \multicolumn{7}{c}{$(\%)$} \\
\cline { 2 - 6 } & $1 M$ & $3 M$ & $6 M$ & $9 M$ & $12 M$ & $r$ & P-value & $b$ \\
\hline$n$ & 31 & 41 & 49 & 46 & 42 & & & \\
Overall & 61.3 & 48.8 & 38.8 & 47.8 & 35.7 & 0.797 & 0.107 & -0.353 \\
$n$ & 26 & 33 & 39 & 36 & 34 & & & \\
Male & 57.7 & 42.4 & 35.9 & 41.7 & 35.3 & 0.756 & 0.139 & -0.371 \\
$n$ & 5 & 8 & 10 & 10 & 8 & & & \\
Female & 80.0 & 75.0 & 50.0 & 70.0 & 37.5 & 0.789 & 0.113 & -0.194 \\
\hline
\end{tabular}

Abbreviations: $b$, regression coefficient; $M$, months after surgery; $r$, correlation corfficient.

Table 2 Change of pyuria after an orthotopic sigmoid colon neobladder replacement

(\%)

\begin{tabular}{lcccccccc} 
& $1 M$ & $3 M$ & $6 M$ & $9 M$ & $12 M$ & $r$ & P-value & $b$ \\
\hline$n$ & 31 & 41 & 48 & 46 & 41 & & & \\
Overall & 74.2 & 43.9 & 33.3 & 21.7 & 22.0 & 0.894 & 0.041 & -0.183 \\
$n$ & 26 & 33 & 38 & 36 & 33 & & & \\
Male & 73.1 & 45.5 & 31.6 & 27.8 & 24.2 & 0.886 & 0.046 & -0.197 \\
$n$ & 5 & 8 & 10 & 10 & 8 & & & \\
Female & 80.0 & 50.0 & 40.0 & 0.0 & 12.5 & 0.912 & 0.031 & -0.128
\end{tabular}

Abbreviations: $b$, regression coefficient; $M$, months after surgery; $r$, correlation corfficient. Bold values indicate statistical significance.
Of 209 for bacteriuria and 207 for pyuria urine samples with evaluable data, $95(45.5 \%)$ were positive for bacteriuria and 76 (36.7\%) had pyuria (10 or more WBCs per h.p.f.). In detail, bacteriuria with $1+$ was seen in $49(23.4 \%), 2+$ in $24(11.5 \%)$ and $3+$ in $20(10.5 \%)$ cases. Pyuria with $10-19$ WBCs per h.p.f. was seen in 24 (11.6\%), 13 (6.28\%) patients with 20-29 WBCs per h.p.f., $12(5.80 \%)$ patients with 30-49 WBCs per h.p.f., 16 (7.73\%) patients with 50-99 WBCs per h.p.f. and $12(5.80 \%)$ patients had 100 or more WBCs per h.p.f.

The change of incidence of bacteriuria and pyuria over the time course after surgery $(1,3,6,9$ and 12 months after surgery) showed that pyuria significantly decreased over time $(P=0.041$ in all patients, 0.046 in males and 0.031 in females) but bacteriuria did not $(P=0.107$ in all patients, 0.139 in males and 0.113 in females). Also, there was no significant change in the occurrence of bacteriuria over the time course after surgery $(P>0.05$; Tables 1 and 2$)$.

Thirty urine cultures from UTI showed positive culture results after surgery, and five Klebisiella pneumoniae, three Enterococcus faecalis, three Enterococcus faecium, two Escherichia coli and two Staphylococcos aureus strains were representatively isolated. Positive urine cultures were shown in nine cases at 1 month, six cases at 3 months, six cases at 6 months, seven cases at 9 months and two cases at 12 months after surgery and these changes were not statistically significant $(P>0.05$; Table 3$)$. In them, there were four cases (7.14\%) of bacteremia and six cases of bacteria $(20.0 \%)$ with antibiotic-resistant strains, which were not susceptible to two or more kinds of antibiotics in beta-lactams, aminoglycosides and fluoroquinolones. In detail, bacteremia cases were caused by two cases of $K$. pneumoniae, one case of $P$. aeruginosa, E. faecium and S. aureus, respectively, and antibiotic-resistant strain of cases were from three cases of methicillin-resistant $S$. aureus, two cases of E. faecium and one case of Corynebacterium spp. The treatments for UTI and bacteremia were performed in 17 cases $(77.3 \%)$ and the antibiotics used representatively were levofloxacin in five cases, tazobactam/piperacillin in three cases, and sulfamethoxazole/trimethoprim and cefepime, meropenem in two cases, respectively.

There was no significant difference between females and males in the occurrences of, or changes in, bacteriuria, pyuria and positive bacterial culture over the time course after surgery $(P>0.05)$.

\section{DISCUSSION}

In terms of the patients with urinary diversion, it is controversial whether asymptomatic bacteriuria or bacterial colonization should be treated or not, ${ }^{8}$ partly because of the emergence of resistant strains caused by possibly unnecessary use of antibiotics or inappropriate dosing. ${ }^{9,10}$ On the other hand, Geerlings et al. reported that untreated bacteriuria and pyuria were risk factors for UTI, especially complicated UTI, including compromised hosts who have diabetes

Table 3 Change of positive urine culture after a sigmoid colon neobladder replacement

\begin{tabular}{|c|c|c|c|c|c|c|c|c|c|}
\hline $1 M(\mathrm{n}=9)$ & & $3 M(\mathrm{n}=6)$ & & $6 M(\mathrm{n}=6)$ & & $9 M(\mathrm{n}=7)$ & & $12 M(n=2)$ & \\
\hline K. pneumoniae & 2 & E. faecium & 2 & MRSA & 1 & E. coli & 1 & K. pneumoniae & 1 \\
\hline Corynebacterium spp. & 1 & $P$. aeruginosa & 1 & M. morgaii & 1 & E. faecium & 1 & E. faecalis & 1 \\
\hline Streptococcus agalactiae & 1 & S. aureus & 1 & K. pneumoniae & 1 & M. morgaii & 1 & & \\
\hline E. faecalis & 1 & K. pneumoniae & 1 & E. coli & 1 & S. aureus & 1 & & \\
\hline M.morgaii & 1 & MRSA & 1 & Protenus penneri & 1 & E. faecalis & 1 & & \\
\hline Serratia marcescens & 1 & & & E. faecalis & 1 & E. avium & 1 & & \\
\hline$P$. aeruginosa & 1 & & & & & Alcaligenes spp. & 1 & & \\
\hline E. coli & 1 & & & & & & & & \\
\hline
\end{tabular}

Abbreviations: M, months after surgery; MRSA, methicillin-resistant Staphylococcus aureus. 
mellitus or have received chemotherapy or steroids. ${ }^{11}$ In the urological field, urinary diversion is often performed after cystectomy, most often for invasive bladder cancer, ${ }^{12}$ and small intestine or colon are often used for this purpose. ${ }^{13}$ From the viewpoint of bacterial flora, the differences between small intestine and colon may influence the causative bacteria in UTI. Wullt et al. ${ }^{6}$ stated that small intestine often had Prevotella species, Peptostreptococcus species, E. coli as the causative bacteria but colon often had Streptococci, Ureoplasma ureolyticum and E. faecalis as causative bacteria. In our sigmoid colon cases, $K$. pneumoniae, E. faecalis, E. faecium and $S$. aureus were representatively often isolated similar to previous reports in the literature. ${ }^{14}$

Once UTI occurs after small intestine or colon neobladder replacement, repeated UTI may cause renal dysfunction or neobladder dysfunctions affecting neobladder compliance or overactivity, or urethral stenosis after catheterization if necessary. ${ }^{15}$ One possible cause for neobladder overactivity may be chronic bacterial colonization or inflammation. Our data showed that the occurrence of postoperative pyuria significantly decreased along with a trend of decreasing positive urine cultures in the 12 months after surgery. This might reflect a potential correlation between pyuria, but not bacteriuria, and positive urine culture over a comparatively long postsurgical observation period even though the direct comparison is not easy to perform. Turan et al. ${ }^{16}$ showed that pyuria, not bacteriuria, was a significant risk factor for UTI. On the other hand, the European Urological Association (EAU) guideline for UTI states that the presence of bacteriuria is needed to diagnose cystitis. ${ }^{17}$ There are no definitive guidelines for UTI in bladder replacement cases and the number of cases studied so far is not enough to draw definitive conclusions. ${ }^{18}$

Regarding the UTI cases with positive urine culture, in our followup for 1 year had 30 cases of positive urine cultures and no significant trend of transition of isolated ratio in a year. Importantly, there were four cases of bacteremia and six bacteria $(20.0 \%)$ with antibioticresistant strains. To our knowledge, no report exists regarding UTI after sigmoid colon neobladder replacements. Our data showed representative bacteremia cases were caused from $K$. pneumoniae and antibiotic-resistant strain cases were representatively from E. faecium or methicillin-resistant $S$. aureus even though statistical evaluation may not be easy for small number of cases. These trends should be paid attention for considering the UTI treatments after sigmoid colon neobladder replacement.

We would like to emphasize the study limitations. First, this is a retrospective study, so the urine culture tests were not performed in all patients but in UTI cases only. Second, the number of patients was not enough to draw definitive conclusion. Third, data on residual urine volumes were lacking and so the analysis could not take into account the presence or absence of residual urine. These limitations will be overcome by our future prospective studies.
In conclusion, bacteriuria and pyuria are very common findings in patients with an orthotopic sigmoid colon neobladder and we found that pyuria decreased significantly over time in the year after surgery. Representative isolated bacteria were K. pneumoniae, E. coli, S. aureus and Enterococcus spp. and included bacteremia cases and antibioticresistant strains. These findings suggest that physicians taking care of sigmoid colon neobladder patients need to be aware these high ratios of bacteriuria, pyuria and UTI including bacteremia.

\section{CONFLICT OF INTEREST}

The authors declare no conflict of interest.

\section{ACKNOWLEDGEMENTS}

We thank Drs Kayo Osawa and Fukashi Yamamichi for statistical analyses and paper drafting, respectively.

1 Wood, D. P. Jr, Montie, J. E., Maatman, T. J. \& Beck, G. J. Radical cystectomy for carcinoma of the bladder in the elderly patient. J. Urol. 138, 46-48 (1987).

2 Skinner, D. G. et al. 25-Year experience in the management of invasive bladder cancer by radical cystectomy. Eur. Urol. 33, S25-S26 (1998).

3 Pagano, F. et al. Vescica Ileale Padovana: a technique for total bladder replacement. Eur. Urol. 17, 149-154 (1990).

4 Skinner, D. G., Lieskovsky, G. \& Boyd, S. Continent urinary diversion. J. Urol. 141, 1323-1327 (1989).

5 Suriano, F. et al. Bacteriuria in patients with an orthotopic ileal neobladder: urinary tract infection or asymptomatic bacteriuria? BJU. Int. 101, 1576-1579 (2008).

6 Wullt, B. et al. Microbial flora in ileal and colonic neobladders. Eur. Urol. 45, 233-239 (2004).

7 Urine cultures In Microbilogy. Complicated UTIs due to Urological Disorders (http:// www.antimicrobe.org/new/printout/e4printout/e4complicated.htm)

8 Akerlund, S., Campanello, M., Kaijser, B. \& Jonsson, O. Bacteriuria in patients with a continent ileal reservoir for urinary diversion does not regularly require antibiotic treatment. Br. J. Urol. 74, 177-181 (1994).

9 Falagas, M. E. \& Vergidis, P. I. Urinary tract infections in patients with urinary diversion. Am. J. Kidney Dis. 46, 1030-1037 (2005).

10 Wullt, B., Agace, W. \& Mansson, W. Bladder, bowel and bugs-bacteriuria in patients with intestinal urinary diversion. World J. Urol. 22, 186-195 (2004).

11 Geerlings, S. E. Urinary tract infections in patients with diabetes mellitus: epidemiology, pathogenesis and treatment. Int. J. Antimicrob. Agents 31, S54-S57 (2008).

12 Khalil, el.-S. A. Long term complications following ileal conduit urinary diversion after radical cystectomy. J. Egypt. Natl Canc. Inst. 22, 13-18 (2010).

13 Pycha, A. et al. Comparison of complications in three incontinent urinary diversions. Eur. Urol. 54, 825-832 (2008).

14 Nakano, Y. et al. Elevated urinary cytokine levels in patients undergoing ileal neobladder replacement compared with sigmoid neobladder replacement. Int. Urol. Nephrol. 39, 467-472 (2007).

15 Hautmann, R. E., de Petriconi, R. C. \& Volkmer, B. G. 25 years of experience with 1,000 neobladders: long-term complications. J. Urol. 185, 2207-2212 (2011).

16 Turan, H. et al. Frequency, risk factors, and responsible pathogenic microorganisms of asymptomatic bacteriuria in patients with type 2 diabetes mellitus. Jpn. J. Infect. Dis. 61, 236-238 (2008).

17 Naber, K. G. et al. EAU guidelines for the management of urinary and male genital tract infections. Urinary Tract Infection (UTI) Working Group of the Health Care Office (HCO) of the European Association of Urology (EAU). Eur. Urol. 40, 576-588 (2001).

18 Obara, W. et al. Eight year experience with Studer ileal neobladder. Jpn. J. Clin. Oncol. 36, 418-424 (2006). 